\title{
Parabolic temperature profile and second-order temperature jump of a slightly rarefied gas in an unsteady two-surface problem
}

\section{$\operatorname{AUTHOR}(\mathrm{S}):$}

Takata, Shigeru; Aoki, Kazuo; Hattori, Masanari; Hadjiconstantinou, Nicolas G.

\section{CITATION:}

Takata, Shigeru ... [et al]. Parabolic temperature profile and second-order temperature jump of a slightly rarefied gas in an unsteady two-surface problem. PHYSICS OF FLUIDS 2012, 24(3): 032002.

\section{ISSUE DATE:}

2012-03

URL:

http://hdl.handle.net/2433/160670

\section{RIGHT:}

Copyright 2012 American Institute of Physics. This article may be downloaded for personal use only. Any other use requires prior permission of the author and the American Institute of Physics. The following article appeared in PHYSICS OF FLUIDS 24, 032002 (2012) and may be found at http://link.aip.org/link/?phf/24/032002 


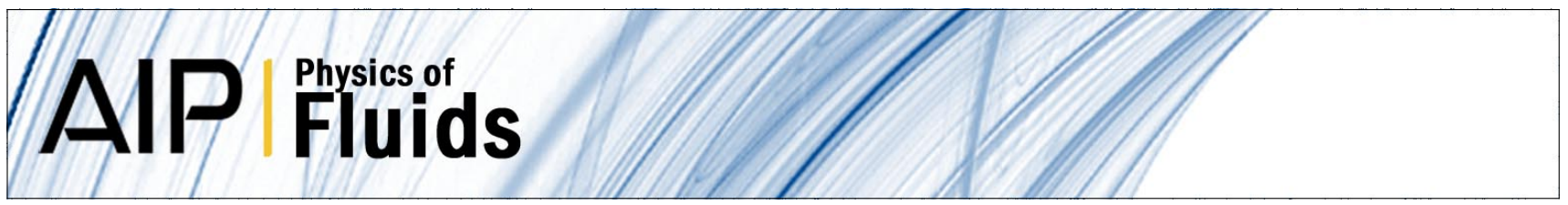

\section{Parabolic temperature profile and second-order temperature jump of a slightly rarefied gas in an unsteady two-surface problem}

Shigeru Takata, Kazuo Aoki, Masanari Hattori, and Nicolas G. Hadjiconstantinou

Citation: Phys. Fluids 24, 032002 (2012); doi: 10.1063/1.3691262

View online: http://dx.doi.org/10.1063/1.3691262

View Table of Contents: http://pof.aip.org/resource/1/PHFLE6/v24/i3

Published by the American Institute of Physics.

\section{Related Articles}

The contribution of diffusion to gas microflow: An experimental study

Phys. Fluids 24, 082004 (2012)

Slip or not slip? A methodical examination of the interface formation model using two-dimensional droplet spreading on a horizontal planar substrate as a prototype system

Phys. Fluids 24, 082105 (2012)

Response to "Comment on 'Velocity slip coefficients based on the hard-sphere Boltzmann equation'” [Phys Fluids 24, 079101 (2012)]

Phys. Fluids 24, 079102 (2012)

Comment on "Velocity slip coefficients based on the hard-sphere Boltzmann equation" [Phys. Fluids 24, 022001 (2012)]

Phys. Fluids 24, 079101 (2012)

Investigation on heat transfer between two coaxial cylinders for measurement of thermal accommodation coefficient

Phys. Fluids 24, 062002 (2012)

\section{Additional information on Phys. Fluids}

Journal Homepage: http://pof.aip.org/

Journal Information: http://pof.aip.org/about/about_the_journal

Top downloads: http://pof.aip.org/features/most_downloaded

Information for Authors: http://pof.aip.org/authors

\section{ADVERTISEMENT}

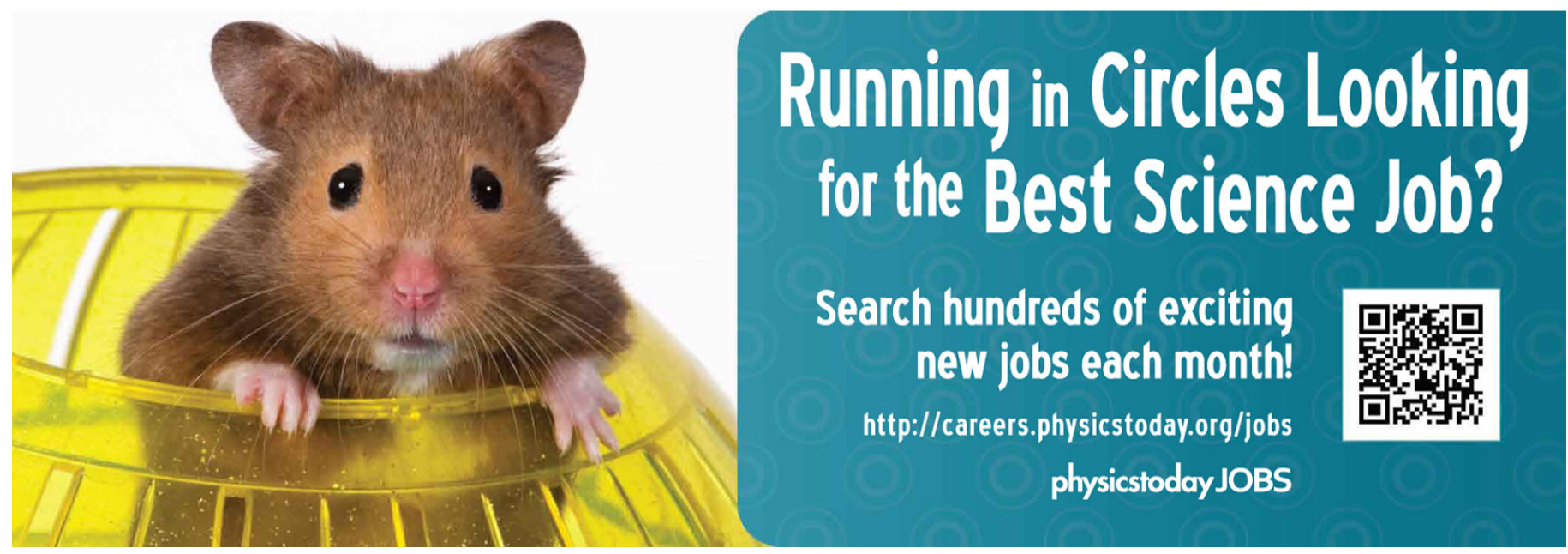




\title{
Parabolic temperature profile and second-order temperature jump of a slightly rarefied gas in an unsteady two-surface problem
}

\author{
Shigeru Takata, ${ }^{1, \text { a) }}$ Kazuo Aoki, ${ }^{1, b)}$ Masanari Hattori, ${ }^{1}$ and \\ Nicolas G. Hadjiconstantinou² \\ ${ }^{1}$ Department of Mechanical Engineering and Sciences, Kyoto University, \\ Kyoto 606-8501, Japan \\ ${ }^{2}$ Department of Mechanical Engineering, Massachusetts Institute of Technology, \\ Cambridge, Massachusetts 02139, USA
}

(Received 17 October 2011; accepted 9 February 2012; published online 6 March 2012)

The behavior of a slightly rarefied monatomic gas between two parallel plates whose temperature grows slowly and linearly in time is investigated on the basis of the kinetic theory of gases. This problem is shown to be equivalent to a boundaryvalue problem of the steady linearized Boltzmann equation describing a rarefied gas subject to constant volumetric heating. The latter has been recently studied by Radtke, Hadjiconstantinou, Takata, and Aoki (RHTA) as a means of extracting the second-order temperature jump coefficient. This correspondence between the two problems gives a natural interpretation to the volumetric heating source and explains why the second-order temperature jump observed by RHTA is not covered by the general theory of slip flow for steady problems. A systematic asymptotic analysis of the time-dependent problem for small Knudsen numbers is carried out and the complete fluid-dynamic description, as well as the related half-space problems that determine the structure of the Knudsen layer and the coefficients of temperature jump, are obtained. Finally, a numerical solution is presented for both the Bhatnagar-GrossKrook model and hard-sphere molecules. The jump coefficient is also calculated by the use of a symmetry relation; excellent agreement is found with the result of the numerical computation. The asymptotic solution and associated second-order jump coefficient obtained in the present paper agree well with the results by RHTA that are obtained by a low variance stochastic method. (c) 2012 American Institute of Physics. [http://dx.doi.org/10.1063/1.3691262]

\section{INTRODUCTION}

The recent development of small-scale devices has renewed interest in microscale or rarefied gas flows. ${ }^{1-6}$ In these flows, the Knudsen number, typically defined as the ratio of the molecular mean free path to the characteristic length scale of the physical system, is no longer negligibly small, signaling that the conventional continuum description, namely the Navier-Stokes equation with the no-slip boundary condition (NS system), does not apply. In such cases, a kinetic approach (Boltzmann system) is appropriate and is typically used.

The connection between the Boltzmann and the NS systems has been studied since the days of Hilbert, and a number of useful results have been obtained in the limit of small Knudsen numbers. ${ }^{7-16}$ Specifically, fluid-dynamic-type sets of equations and appropriate slip and jump boundary conditions for describing the steady gas behavior in the regime of small Knudsen numbers (the so-called slip

\footnotetext{
a) Also at Advanced Research Institute of Fluid Science and Engineering, Kyoto University, Kyoto 606-8501, Japan. Electronic mail: takata.shigeru.4a@kyoto-u.ac.jp.

b) Also at Advanced Research Institute of Fluid Science and Engineering, Kyoto University, Kyoto 606-8501, Japan.
} 


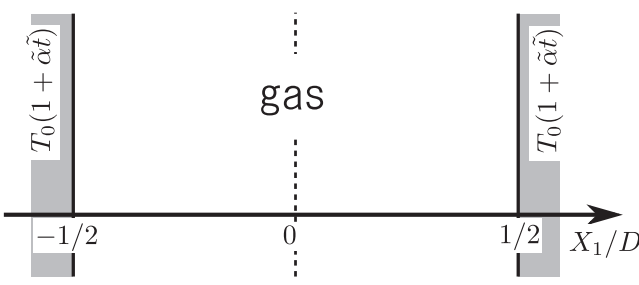

FIG. 1. Sketch of the problem.

flow regime) have been established ${ }^{11-13}$ since the late 1960s and early 1970s. For rigorous and complete descriptions of the general theory of slip flow, the reader is referred to Refs. 2 and 6. In the present paper, we shed light on a new feature of the jump of temperature that can occur at the second order of the Knudsen number in unsteady problems. The present work is motivated by the simulation results recently obtained by Radtke, Hadjiconstantinou, Takata, and Aoki (RHTA), which will be reported in a separate paper (see also Chap. 7 of Ref. 17), and is intended to contribute to a growing research topic on the second-order slip (e.g., Refs. 3 and 18-22).

Using a low variance stochastic method, ${ }^{23-26}$ RHTA carried out numerical simulations of a slightly rarefied gas bounded by two parallel walls subject to constant volumetric heating. The parabolic temperature profile associated with the volumetric heating allowed them to evaluate the second-order temperature-jump coefficient, which, in the case of the Bhatnagar-Gross-Krook (BGK) [or Boltzmann-Krook-Welander (BKW)] model, ${ }^{27,28}$ did not agree with the one predicted (coefficient $d_{3}$ in Sec. 3.1.5 of Ref. 6) by the general theory of slip flow for steady problems (Refs. 2,6, and 11).

In order to explain the above observation, in the present paper, we consider a slightly rarefied gas between two parallel plates whose temperature grows slowly and linearly in time which, as we show in Sec. II, can be reduced to the problem studied by RHTA. We subsequently investigate the behavior of the gas by a systematic asymptotic analysis for small Knudsen numbers (Secs. III and IV). Our results show that, as expected, the reduced half-space problem that determines the second-order jump of temperature is new and does not appear in the theory for steady problems. We also perform numerical computations to determine the second-order temperature-jump coefficient and the structure of the related Knudsen layer for the BGK model and for hard-sphere molecules (Sec. V). The solution is compared, not only in the fluid-dynamic region but also in the Knudsen-layer, with the simulation results that RHTA have obtained by the low variance stochastic method.

\section{PROBLEM}

Consider a rarefied monatomic gas between two parallel plates located at $X_{1}= \pm D / 2$ and kept at the same uniform temperature $T_{w}$, where $X_{i}$ is the Cartesian space coordinate (Fig. 1). The temperature $T_{w}$ is uniform on the plates and grows slowly and linearly in time $\tilde{t}$, i.e., $T_{w}=T_{0}(1+\tilde{\alpha} \tilde{t})$, where $T_{0}$ is a reference temperature and $\tilde{\alpha}$ is a small constant. We will investigate the behavior of this gas after a long time has passed from some initial state under the following assumptions: (i) The behavior of the gas is described by the Boltzmann equation (or its model equation such as the BGK model). (ii) The gas molecules are diffusely reflected on the plates. (iii) The change of plate temperature during the characteristic time of heat conduction is small, i.e., $\alpha \equiv \tilde{\alpha} t_{0} \ll 1$ with $t_{0}$ $\sim \rho_{0} R D^{2} / \lambda$, and thus the equation and boundary condition can be linearized around the reference equilibrium state at rest with density $\rho_{0}$ and temperature $T_{0}$. Here, $\rho_{0}$ is the average density of the gas, $\lambda$ is the thermal conductivity, and $R$ is the specific gas constant, i.e., $R=k / m$ with $k$ denoting the Boltzmann constant and $m$ the mass of a molecule. (iv) The mean free path $\ell_{0}$ of a molecule at the reference state is much shorter than the distance between the plates $D$, or, in other words, the Knudsen number $\mathrm{Kn}=\ell_{0} / D$ is small. Hereinafter, we set the time scale $t_{0}$ as $t_{0}=D^{2} / \ell_{0}\left(\pi R T_{0} / 2\right)^{1 / 2}$.

Let us denote the time $\tilde{t}$ by $t_{0} t$, the position $X_{1}$ by $D x$, the molecular velocity by $\left(2 R T_{0}\right)^{1 / 2} \zeta$, and the velocity distribution function by $\rho_{0}\left(2 R T_{0}\right)^{-3 / 2}\left[1+\alpha t\left(|\zeta|^{2}-\frac{3}{2}\right)+\phi\right] E$, where $E$ is the normalized Maxwellian defined by $E(|\zeta|)=\pi^{-3 / 2} \exp \left(-|\zeta|^{2}\right.$ ). By assumption (iii), $\phi$ is a small 
quantity and its higher order contributions will be neglected. Then, $\phi$ can be sought as a function of $x$ and $\zeta$ that is even both in $\zeta_{2}$ and $\zeta_{3}$ and satisfies the following (dimensionless) steady inhomogeneous linearized Boltzmann equation and the boundary condition:

$$
\begin{gathered}
\zeta_{1} \partial_{x} \phi=\frac{1}{\varepsilon} \mathcal{L}[\phi]-\varepsilon \alpha\left(|\zeta|^{2}-\frac{3}{2}\right), \\
\phi= \pm 2 \sqrt{\pi} \int_{\zeta_{1} \gtrless 0} \zeta_{1} \phi E d \zeta, \quad \zeta_{1} \lessgtr 0, x= \pm \frac{1}{2},
\end{gathered}
$$

where $\partial_{x}=\partial / \partial x, \varepsilon=(\sqrt{\pi} / 2) \mathrm{Kn}, \mathcal{L}$ is the linearized collision operator, and $\boldsymbol{d} \zeta=d \zeta_{1} d \zeta_{2} d \zeta_{3}$.

For later convenience, we define the macroscopic quantities and their notation here. The density, flow velocity, temperature, and pressure of the gas are given by $\rho_{0}(1+\omega),\left(2 R T_{0}\right)^{1 / 2}(u, 0,0), T_{0}(1$ $+\alpha t+\tau)$, and $p_{0}(1+\alpha t+P)$, where $p_{0}=\rho_{0} R T_{0}$, while $\omega, u, \tau$, and $P$ are defined by

$$
\omega=\langle\phi\rangle, u=\left\langle\zeta_{1} \phi\right\rangle, \tau=\frac{2}{3}\left\langle\left(|\zeta|^{2}-\frac{3}{2}\right) \phi\right\rangle, P=\frac{2}{3}\left\langle|\zeta|^{2} \phi\right\rangle,
$$

and the angle brackets denote the following moment:

$$
\langle\Phi\rangle=\int \Phi(x, \zeta) E(|\zeta|) d \zeta .
$$

Note that $\tau$ (more precisely $T_{0} \tau$ ) is a perturbation of the temperature from the plate temperature. By definition, $P=\omega+\tau$.

It should be noted that problem (1) is equivalent to the constant volumetric heating problem studied by RHTA. The present formulation shows that the parabolic temperature profile obtained in RHTA via a source term in the governing (Boltzmann) equation can be naturally obtained (no additional source term) by wall temperatures following the time dependence described above. Therefore, as we show below, the temperature jump observed here and in the work by RHTA is not covered by the general theory of slip flow ${ }^{2,6,11}$ for steady problems.

The volumetric heating term used in RHTA, $-\epsilon \alpha\left(|\zeta|^{2}-\frac{3}{2}\right)$, leads to a spatially and temporally constant increase in the gas thermal energy. This heating (or cooling when $\alpha>0$ ) is similar to the cooling of the granular gas in that it conserves mass and momentum. However, it is independent of the gas state, in contrast to the granular gas case. It should also be noted that no essential difference between heating $(\alpha<0)$ and cooling $(\alpha>0)$ exists in the case of RHTA. The constant volumetric heating (cooling) implies a controlled energy supply (loss) in the gas, which is balanced by the loss (supply) of energy through the side walls through the heat conduction. In the present time-dependent problem, the temperature profile is determined by the interplay between the gas inertia and the energy supply (loss) due to the change of wall temperature. Our formulation shows that, when measured relative to the time-varying wall temperature, the temperature profile is identical to the heat addition (loss) case of RHTA.

Because of assumption (iv), $\varepsilon$ is a small constant. We will investigate the problem (1) by a systematic asymptotic analysis for small $\varepsilon$, following Sone's method. ${ }^{2,6,11}$

\section{HILBERT SOLUTION}

Putting aside the boundary condition, we seek a moderately varying solution $\phi_{H}$ (the Hilbert solution) in a power series of $\varepsilon$ :

$$
\phi_{H}=\phi_{H 0}+\phi_{H 1} \varepsilon+\phi_{H 2} \varepsilon^{2}+\cdots
$$

Corresponding macroscopic quantities, which we denote by $h_{H}(h=P, u, \tau, \omega)$, are also expanded as

$$
h_{H}=h_{H 0}+h_{H 1} \varepsilon+h_{H 2} \varepsilon^{2}+\cdots,
$$


where $h_{H m}(m=0,1, \ldots)$ is defined by Eq. (2) with $\phi$ being replaced by $\phi_{H m}$. Substitution of (3) into (1a) leads to

$$
\begin{aligned}
\mathcal{L}\left[\phi_{H 0}\right] & =0, \\
\mathcal{L}\left[\phi_{H 1}\right] & =\zeta_{1} \partial_{x} \phi_{H 0}, \\
\mathcal{L}\left[\phi_{H 2}\right] & =\zeta_{1} \partial_{x} \phi_{H 1}+\alpha\left(|\zeta|^{2}-\frac{3}{2}\right), \\
\mathcal{L}\left[\phi_{H m}\right] & =\zeta_{1} \partial_{x} \phi_{H m-1},(m \geq 3) .
\end{aligned}
$$

This is a set of linear integral equations and can be solved from the lowest order, provided that the following condition is satisfied:

$$
\left\langle\left(\begin{array}{c}
1 \\
\zeta_{1} \\
|\zeta|^{2}
\end{array}\right) \times \text { R.H.S. of Eq. }(5)\right\rangle=0,
$$

because $\mathcal{L}[f]=0$ has nontrivial solutions $f=1, \zeta_{1}, \zeta_{2}, \zeta_{3},|\zeta|^{2}$ (the so-called collision invariants).

\section{A. Outline of the analysis}

The solvability condition (6) for Eq. (5a) is automatically satisfied, yielding

$$
\phi_{H 0}=P_{H 0}+2 \zeta_{1} u_{H 0}+\left(|\zeta|^{2}-\frac{5}{2}\right) \tau_{H 0} .
$$

Let us proceed to Eq. (5b). By the substitution of (7), the solvability condition (6) is rewritten as

$$
\frac{d u_{H 0}}{d x}=0, \frac{d P_{H 0}}{d x}=0 .
$$

Thus, Eq. (5b) is reduced to

$$
\mathcal{L}\left[\phi_{H 1}\right]=\zeta_{1}\left(|\zeta|^{2}-\frac{5}{2}\right) \frac{d \tau_{H 0}}{d x},
$$

yielding

$$
\phi_{H 1}=P_{H 1}+2 \zeta_{1} u_{H 1}+\left(|\zeta|^{2}-\frac{5}{2}\right) \tau_{H 1}-\zeta_{1} A \frac{d \tau_{H 0}}{d x} .
$$

Here $A$ is a function of $|\zeta|$ defined by

$$
\mathcal{L}\left[\zeta_{1} A\right]=-\zeta_{1}\left(|\zeta|^{2}-\frac{5}{2}\right) \quad \text { with }\left\langle|\zeta|^{2} A\right\rangle=0 .
$$

Next, we consider Eq. (5c). With the aid of Eq. (8), the solvability condition (6) can be rewritten as

$$
\begin{gathered}
\frac{d u_{H 1}}{d x}=0, \frac{d P_{H 1}}{d x}=0, \\
-\frac{5}{4} \gamma_{2} \frac{d^{2} \tau_{H 0}}{d x^{2}}+\frac{3}{2} \alpha=0,
\end{gathered}
$$

where $\gamma_{2}$ is a constant (namely, the dimensionless thermal conductivity) defined by $\gamma_{2}=2 I_{6}(A)$ with

$$
I_{n}(X)=\frac{2}{15}\left\langle|\zeta|^{n-2} X\right\rangle
$$


Thus, Eq. (5c) is reduced to

$$
\mathcal{L}\left[\phi_{H 2}\right]=\zeta_{1}\left(|\zeta|^{2}-\frac{5}{2}\right) \frac{d \tau_{H 1}}{d x}-\frac{6}{5} \frac{\alpha}{\gamma_{2}}\left(\zeta_{1}^{2}-\frac{1}{3}|\zeta|^{2}\right) A+\frac{6}{5} \frac{\alpha}{\gamma_{2}}\left[\frac{5}{6} \gamma_{2}\left(|\zeta|^{2}-\frac{3}{2}\right)-\frac{1}{3}|\zeta|^{2} A\right],
$$

whose solution is

$$
\phi_{H 2}=P_{H 2}+2 \zeta_{1} u_{H 2}+\left(|\zeta|^{2}-\frac{5}{2}\right) \tau_{H 2}-\zeta_{1} A \frac{d \tau_{H 1}}{d x}-\frac{6}{5} \frac{\alpha}{\gamma_{2}}\left(\zeta_{1}^{2}-\frac{1}{3}|\zeta|^{2}\right) F-\frac{6}{5} \frac{\alpha}{\gamma_{2}} F_{d} .
$$

Here, $F$ and $F_{d}$ are functions of $|\zeta|$ defined by

$$
\begin{aligned}
& \mathcal{L}\left[\zeta_{1} \zeta_{2} F\right]=\zeta_{1} \zeta_{2} A, \\
& \mathcal{L}\left[F_{d}\right]=-\frac{5}{6} \gamma_{2}\left(|\zeta|^{2}-\frac{3}{2}\right)+\frac{1}{3}|\zeta|^{2} A \quad \text { with }\left\langle F_{d}\right\rangle=0 \text { and }\left\langle|\zeta|^{2} F_{d}\right\rangle=0 .
\end{aligned}
$$

We proceed to Eq. (5d) with $m=3$. With the aid of Eqs. (8) and (10), the solvability condition (6) can be rewritten as

$$
\begin{aligned}
\frac{d u_{H 2}}{d x} & =0, \frac{d P_{H 2}}{d x}=0, \\
\frac{d^{2} \tau_{H 1}}{d x^{2}} & =0 .
\end{aligned}
$$

Then, Eq. (5d) simplifies to

$$
\mathcal{L}\left[\phi_{H 3}\right]=\zeta_{1}\left(|\zeta|^{2}-\frac{5}{2}\right) \frac{d \tau_{H 2}}{d x}
$$

yielding

$$
\phi_{H 3}=P_{H 3}+2 \zeta_{1} u_{H 3}+\left(|\zeta|^{2}-\frac{5}{2}\right) \tau_{H 3}-\zeta_{1} A \frac{d \tau_{H 2}}{d x}
$$

Finally, the substitution of this expression reduces the solvability condition (6) for Eq. (5d) with $m=4$ to

$$
\begin{aligned}
& \frac{d u_{H 3}}{d x}=0, \frac{d P_{H 3}}{d x}=0, \\
& \frac{d^{2} \tau_{H 2}}{d x^{2}}=0 .
\end{aligned}
$$

If we proceed further, we will obtain for any $m \geq 4$

$$
\phi_{H m}=P_{H m}+2 \zeta_{1} u_{H m}+\left(|\zeta|^{2}-\frac{5}{2}\right) \tau_{H m}-\zeta_{1} A \frac{d \tau_{H m-1}}{d x},
$$

and

$$
\frac{d u_{H m}}{d x}=0, \frac{d P_{H m}}{d x}=0, \frac{d^{2} \tau_{H m-1}}{d x^{2}}=0 .
$$

\section{B. Summary}

In Sec. III A, we have obtained a set of fluid-dynamic-type equations for the macroscopic quantities, namely, Eqs. (8) and (10b) for $O\left(\varepsilon^{0}\right)$; Eqs. (10a) and (12b) for $O\left(\varepsilon^{1}\right)$; and Eqs. (12a) and (14b) for $O\left(\varepsilon^{2}\right)$, and so on.

Since $\phi_{H 0}$ is a linearized Maxwellian, it satisfies the boundary condition (1b), if we set

$$
u_{H 0}=0, \tau_{H 0}=0 \quad \text { on } x= \pm \frac{1}{2} .
$$


Note that the reference density $\rho_{0}$ is the average density between the plates, so that the average of perturbed density $\omega$, which is related to $P$ and $\tau$ by $\omega=P-\tau$, should vanish. Thus, the quantities of $O\left(\varepsilon^{0}\right)$ are obtained from Eqs. (8), (10b), and (17) as

$$
\begin{gathered}
u_{H 0}=0, \omega_{H 0}=-\frac{3 \alpha}{5 \gamma_{2}}\left(x^{2}-\frac{1}{12}\right), \\
\tau_{H 0}=-\frac{3 \alpha}{5 \gamma_{2}}\left(\frac{1}{4}-x^{2}\right), P_{H 0}=-\frac{\alpha}{10 \gamma_{2}} .
\end{gathered}
$$

The above $\tau_{H 0}$ shows that $\phi_{H 1}$ is no longer a linearized Maxwellian [see Eq. (9)] and does not satisfy the boundary condition (1b). The same is true for $\phi_{H 2}$ [see Eq. (11)]. Thus, by the analysis in Sec. III A, we cannot determine the gas behavior completely. What we can see from Eqs. (10a) and (12b) and from Eqs. (12a) and (14b) is that

$$
\begin{array}{lll}
P_{H 1}=c_{1}, & u_{H 1}=c_{2}, & \tau_{H 1}=c_{3}, \\
P_{H 2}=c_{4}, & u_{H 2}=c_{5}, & \tau_{H 2}=c_{6},
\end{array}
$$

where $c_{i}(i=1,2, \ldots, 6)$ are (undetermined) constants. Note that the linear dependence of temperature in $x$ vanishes because the temperature should be symmetric in $x$ with respect to $x=0$. In order to find the solution that satisfies the boundary condition, in Sec. IV, we will introduce a correction in the microscopic boundary-layer (the Knudsen-layer correction) to the Hilbert solution. The analysis of the correction will determine the constants $c_{i}$ and the structure of the Knudsen layer. If $c_{3}$ and $c_{6}$ are nonzero, a jump of temperature at the first- and second-orders of $\varepsilon$ exists.

Thanks to the symmetric temperature profile in $x$, all of $\tau_{H m}, P_{H m}$, and $u_{H m}$ for $m \geq 3$ are found to be constant from Eqs. (14a) and (16), and thus the corresponding $\phi_{H m}$ are reduced to linearized Maxwellians [see Eqs. (13) and (15)]. Therefore, if we set $u_{H m}=\tau_{H m}=0, \phi_{H m}$ satisfies the boundary condition (1b) at $O\left(\varepsilon^{m}\right)$. Consequently, the Knudsen-layer correction to be discussed in Sec. IV is not required at the third and higher order of $\varepsilon . P_{H m}$ will be determined again by the constraint that the perturbed density averaged in $x$ should vanish.

\section{KNUDSEN-LAYER CORRECTION AND THE SOLUTION FOR THE ENTIRE GAP}

Because of the symmetry of the problem $\phi\left(x, \zeta_{1}, \cdot\right)=\phi\left(-x,-\zeta_{1}, \cdot\right)$, it is enough to consider the correction near the left plate at $x=-\frac{1}{2}$. The correction near the right plate is its mirror image.

We will obtain the solution $\phi$ in the form of $\phi=\phi_{H}+\phi_{K}$, where $\phi_{K}$ is the Knudsenlayer correction, which is appreciable only in the thin layer adjacent to the left plate [thus, $\partial_{x} \phi_{K}$ $\left.=(1 / \varepsilon) O\left(\phi_{K}\right)\right]$ and is $O(\varepsilon)$ because the correction is not required at the zeroth order. Corresponding correction to macroscopic quantities will be denoted by $h_{K}$, i.e., $h=h_{H}+h_{K}$. We introduce the stretched coordinate $\eta$ which is defined by $\eta=\left(x+\frac{1}{2}\right) / \varepsilon$ near the left plate. Then, $\phi_{K}$ is the solution of the following problem:

$$
\begin{aligned}
\zeta_{1} \partial_{\eta} \phi_{K} & =\mathcal{L}\left[\phi_{K}\right] \\
\phi_{K} & =-\phi_{H}-2 \sqrt{\pi} \int_{\zeta_{1}<0} \zeta_{1}\left(\phi_{K}+\phi_{H}\right) E d \zeta, \quad \zeta_{1}>0, \eta=0 \\
\phi_{K} \rightarrow 0 & \text { as } \eta \rightarrow \infty
\end{aligned}
$$

where $\partial_{\eta}=\partial / \partial \eta$. It is seen by taking the moment of Eq. (18a), i.e., 〈Eq. (18a) $\rangle$, that $u_{K} \equiv 0$ because of the condition (18c). Since $u\left(=u_{H}+u_{K}\right)=0$ on the plate, we see that $u_{H}=0$ on the plate and obtain

$$
c_{2}=c_{5}=0 .
$$

Since $\phi_{K}=O(\varepsilon)$, we expand it as $\phi_{K}=\phi_{K 1} \varepsilon+\phi_{K 2} \varepsilon^{2}+\cdots$ and correspondingly $h_{K}$ as $h_{K}$ $=h_{K 1} \varepsilon+h_{K 2} \varepsilon^{2}+\cdots$, where $h_{K m}(m=1,2, \ldots)$ is given by Eq. (2) with $\phi$ being replaced by 
$\phi_{K m}$. Actually, as mentioned in the last paragraph of Sec. III B, the expansion terminates at $O\left(\varepsilon^{2}\right)$ in the present problem. Substitution of the expansion of $\phi_{K}$ into Eq. (18) leads to boundary-value problems for $\phi_{K 1}$ and $\phi_{K 2}$. To be more specific, if we let

$$
\begin{aligned}
& \psi^{(1)}=-\left(\frac{3 \alpha}{5 \gamma_{2}}\right)^{-1} \phi_{K 1}, \psi^{(2)}=\left(\frac{6 \alpha}{5 \gamma_{2}}\right)^{-1} \phi_{K 2}, \\
& \beta^{(1)}=-\left(\frac{3 \alpha}{5 \gamma_{2}}\right)^{-1} c_{3}, \beta^{(2)}=\left(\frac{6 \alpha}{5 \gamma_{2}}\right)^{-1} c_{6},
\end{aligned}
$$

we obtain the following equations and boundary conditions for $\psi^{(i)}$ :

$$
\begin{aligned}
& \zeta_{1} \partial_{\eta} \psi^{(i)}=\mathcal{L}\left[\psi^{(i)}\right], \quad(i=1,2), \\
& \psi^{(i)}=I^{(i)}-2 \sqrt{\pi} \int_{\zeta_{1}<0} \zeta_{1} \psi^{(i)} E \boldsymbol{d} \zeta, \zeta_{1}>0, \eta=0, \\
& \psi^{(i)} \rightarrow 0 \quad \text { as } \eta \rightarrow \infty,
\end{aligned}
$$

where

$$
\begin{aligned}
& I^{(1)}=-\left(|\zeta|^{2}-2\right) \beta^{(1)}+\zeta_{1} A, \\
& I^{(2)}=-\left(|\zeta|^{2}-2\right) \beta^{(2)}-\frac{\sqrt{\pi}}{2}\left(\left\langle|\zeta| F_{d}\right\rangle+\frac{1}{6}\left\langle|\zeta|^{3} F\right\rangle\right)+F_{d}+\left(\zeta_{1}^{2}-\frac{1}{3}|\zeta|^{2}\right) F .
\end{aligned}
$$

The solution of the half-space problem (20) exists uniquely and the constant $\beta^{(1)}$ (or $\beta^{(2)}$ ) is determined uniquely together with the solution $\psi^{(1)}\left(\right.$ or $\left.\psi^{(2)}\right) .{ }^{10,29-31}$

The coefficients $-3 \alpha / 5 \gamma_{2}$ and $6 \alpha / 5 \gamma_{2}$ in Eq. (19) are the first and second derivatives of temperature $\tau_{H}$ at the left plate. Accordingly, $\psi^{(1)}$ (and thus $\phi_{K 1}$ ) is seen to be the classical temperature-jump problem, which has been studied by various authors (e.g., Refs. 28, and 32-35). On the other hand, $\psi^{(2)}$ is the solution of the problem of jump caused by the second derivative of temperature with respect to $x$, which is, however, different from the jump problem caused by the second derivative of temperature predicted by the theory for steady problems.

The fact that the amount of temperature jump caused by the second derivative of temperature is different depending on whether the problem is unsteady or steady may appear to be paradoxical. According to the theory for steady flows (Refs. 2 and 6), the temperature field satisfies the Laplace equation, which reduces to a vanishing second derivative of temperature $\left(d^{2} \tau_{H} / d x^{2}=0\right.$, where this $\tau$ is perturbed temperature from a reference uniform equilibrium state) in one-dimensional problems. In other words, the temperature jump caused by the second derivatives of temperature does not manifest itself in steady one-dimensional problems. In contrast, in the present unsteady onedimensional problem, $\tau_{H}$ satisfies the equation of the form $d^{2} \tau_{H} / d x^{2}=\operatorname{const}(\neq 0)$ [see Eq. (10b)], which is a one-dimensional version of the Poisson equation, not the Laplace equation. As we have seen, the non-zero constant in this equation may be thought of as originating from a volumetric heating source in a steady framework, or, in the unsteady setting, from the time dependence of plate temperature. In other words, the time dependence of the problem introduces a new temperature jump caused by the second derivative of temperature. This difference between steady and unsteady problems is maintained in the general two- and three-dimensional problems, in which the temperature jump associated with the second derivative of temperature also appears in steady problems.

Finally, the constants $c_{1}$ and $c_{4}$ (namely, $P_{H 1}$ and $P_{H 2}$ ) as well as $P_{H m}$ for $m \geq 3$ are determined so as to make the perturbed density averaged across the space between the two plates vanish. This procedure yields the relations $\omega_{H 1}=0, P_{H 1}=c_{3}, \omega_{H 2}=-2 \int_{0}^{\infty} \omega_{K 1} d \eta, P_{H 2}=c_{6}-\omega_{H 2}, \omega_{H 3}$ $=-P_{H 3}=-2 \int_{0}^{\infty} \omega_{K 2} d \eta$, and $\omega_{H m}=P_{H m}=0$ for $m \geq 4$, thus they are completely determined after solving Eq. (20).

To summarize, the macroscopic quantities are expressed for the entire gap between the plates by the sum of $h=h_{H 0}+\left(h_{H 1}+h_{K 1}\right) \varepsilon+\left(h_{H 2}+h_{K 2}\right) \varepsilon^{2}+h_{H 3} \varepsilon^{3}+\cdots$, where $h_{H m}$ for $m \geq 3$ vanishes 
except for $\omega_{H 3}$ and $P_{H 3}$. We have obtained the following asymptotic solutions of $u, \tau, \omega$, and $P$ :

$$
u=0,
$$

$$
\begin{aligned}
\tau= & -\frac{3 \alpha}{5 \gamma_{2}}\left\{\left(\frac{1}{4}-x^{2}\right)+\left[\beta^{(1)}+\Theta^{(1)}\left(\eta_{-}\right)+\Theta^{(1)}\left(\eta_{+}\right)\right] \varepsilon\right. \\
& \left.-2\left[\beta^{(2)}+\Theta^{(2)}\left(\eta_{-}\right)+\Theta^{(2)}\left(\eta_{+}\right)\right] \varepsilon^{2}\right\} \\
\omega= & -\frac{3 \alpha}{5 \gamma_{2}}\left\{x^{2}-\frac{1}{12}+\left[\Omega^{(1)}\left(\eta_{-}\right)+\Omega^{(1)}\left(\eta_{+}\right)\right] \varepsilon\right. \\
& \left.-2\left[\int_{0}^{\infty} \Omega^{(1)} d \eta+\Omega^{(2)}\left(\eta_{-}\right)+\Omega^{(2)}\left(\eta_{+}\right)\right] \varepsilon^{2}+4 \int_{0}^{\infty} \Omega^{(2)} d \eta \varepsilon^{3}\right\},
\end{aligned}
$$

$$
P=\omega+\tau
$$

where

$$
\begin{aligned}
& \Omega^{(i)}=\left\langle\psi^{(i)}\right\rangle, \Theta^{(i)}=\frac{2}{3}\left\langle\left(|\zeta|^{2}-\frac{3}{2}\right) \psi^{(i)}\right\rangle, \\
& \eta_{+}=\frac{1}{\varepsilon}\left(-x+\frac{1}{2}\right), \eta_{-}=\frac{1}{\varepsilon}\left(x+\frac{1}{2}\right) .
\end{aligned}
$$

\section{NUMERICAL DATA}

Because the problem for $\psi^{(1)}$ is classical, reliable data for $\beta^{(1)}, \Omega^{(1)}$, and $\Theta^{(1)}$ are available in the literature. For example, $\beta^{(1)}, \Omega^{(1)}$, and $\Theta^{(1)}$ correspond to $d_{1}, \Omega_{1}$, and $\Theta_{1}$ in Ref. 6 , in which data for the BGK (or BKW) model and hard-sphere molecules can be found. $\Omega^{(1)}$ and $\Theta^{(1)}$ are tabulated in Table I for use in Eq. (21) (the data in the table were obtained in Ref. 35; the accuracy would be slightly better than those in Ref. 6). The values of $\beta^{(1)}$ and $\int_{0}^{\infty} \Omega^{(1)} d \eta$ are

$$
\begin{aligned}
& \beta^{(1)}=1.30272(\mathrm{BGK}), 2.4001 \text { (hard sphere), } \\
& \int_{0}^{\infty} \Omega^{(1)} d \eta=0.3230(\mathrm{BGK}), 0.5241 \text { (hard sphere). }
\end{aligned}
$$

On the other hand, the problem for $\psi^{(2)}$ has not been solved, and thus data for $\beta^{(2)}, \Omega^{(2)}$, and $\Theta^{(2)}$ are not available. Data for $F_{d}$ are also not available. In the present section, we provide these data for the BGK model and hard-sphere molecules to complete our analysis. The specific form of the linearized collision integral for these cases is the following: for the BGK model,

$$
\mathcal{L}[\phi]=-\phi+\omega+2 \zeta_{1} u+\left(|\zeta|^{2}-\frac{3}{2}\right) \tau,
$$

and $\ell_{0}=\left(8 R T_{0} / \pi\right)^{1 / 2} / A_{c} \rho_{0}$, where $A_{c}$ is a constant such that $A_{c} \rho_{0}$ is the collision frequency of gas molecules at the reference state; for hard-sphere molecules,

$$
\begin{aligned}
& \mathcal{L}[\phi]=-v(|\zeta|) \phi+\int\left[K_{1}(\boldsymbol{\zeta}, \boldsymbol{\xi})-K_{2}(\boldsymbol{\zeta}, \boldsymbol{\xi})\right] \phi(\xi) d \boldsymbol{\xi} \\
& v(z)=\frac{1}{2 \sqrt{2}}\left[\exp \left(-z^{2}\right)+\left(2 z+\frac{1}{z}\right) \int_{0}^{z} \exp \left(-y^{2}\right) d y\right] \\
& K_{1}(\boldsymbol{\zeta}, \boldsymbol{\xi})=\frac{1}{\sqrt{2} \pi|\zeta-\xi|} \exp \left(-|\boldsymbol{\xi}|^{2}+\frac{|\boldsymbol{\xi} \times \zeta|^{2}}{|\boldsymbol{\xi}-\zeta|^{2}}\right)
\end{aligned}
$$


TABLE I. Knudsen-layer functions $\Omega^{(1)}$ and $\Theta^{(1)}$.

\begin{tabular}{|c|c|c|c|c|c|}
\hline \multicolumn{3}{|c|}{ Hard sphere } & \multicolumn{3}{|c|}{ BGK } \\
\hline$\eta$ & $\Omega^{(1)}$ & $-\Theta^{(1)}$ & $\eta$ & $\Omega^{(1)}$ & $-\Theta^{(1)}$ \\
\hline 0.0000 & 0.5164 & 0.7378 & 0.0000 & 0.3477 & 0.4492 \\
\hline 0.0235 & 0.4710 & 0.6766 & 0.0249 & 0.3130 & 0.4101 \\
\hline 0.0517 & 0.4382 & 0.6314 & 0.0491 & 0.2925 & 0.3860 \\
\hline 0.1089 & 0.3911 & 0.5655 & 0.1289 & 0.2474 & 0.3314 \\
\hline 0.1567 & 0.3615 & 0.5234 & 0.2483 & 0.2049 & 0.2779 \\
\hline 0.2011 & 0.3387 & 0.4906 & 0.4553 & 0.1585 & 0.2175 \\
\hline 0.3141 & 0.2929 & 0.4241 & 0.6091 & 0.1349 & 0.1860 \\
\hline 0.4091 & 0.2631 & 0.3805 & 0.7492 & 0.1181 & 0.1633 \\
\hline 0.6161 & 0.2145 & 0.3085 & 0.9055 & 0.1029 & 0.1425 \\
\hline 0.7584 & 0.1892 & 0.2707 & 1.1462 & 0.0846 & 0.1173 \\
\hline 1.0047 & 0.1552 & 0.2198 & 1.2596 & 0.0776 & 0.1076 \\
\hline 1.1904 & 0.1353 & 0.1899 & 1.4285 & 0.0686 & 0.0950 \\
\hline 1.3925 & 0.1174 & 0.1632 & 1.6207 & 0.0599 & 0.0830 \\
\hline 1.6669 & 0.0979 & 0.1342 & 1.8993 & 0.0498 & 0.0688 \\
\hline 1.8426 & 0.0876 & 0.1190 & 2.0571 & 0.0451 & 0.0621 \\
\hline 2.0259 & 0.0783 & 0.1053 & 2.4552 & 0.0353 & 0.0485 \\
\hline 2.5490 & 0.0576 & 0.0754 & 2.7963 & 0.0290 & 0.0396 \\
\hline 3.0422 & 0.0438 & 0.0559 & 3.1895 & 0.0233 & 0.0316 \\
\hline 3.4872 & 0.0345 & 0.0431 & 3.5835 & 0.0189 & 0.0255 \\
\hline 4.0281 & 0.0261 & 0.0318 & 3.8977 & 0.0161 & 0.0215 \\
\hline 5.0001 & 0.0160 & 0.0188 & 4.3903 & 0.0125 & 0.0167 \\
\hline 6.2778 & 0.0086 & 0.0097 & 5.3111 & 0.0080 & 0.0106 \\
\hline 8.0709 & 0.0037 & 0.0040 & 6.9386 & 0.0039 & 0.0050 \\
\hline 10.0635 & 0.0015 & 0.0015 & 10.0712 & 0.0010 & 0.0013 \\
\hline 16.0206 & 0.0001 & 0.0001 & 16.2166 & 0.0001 & 0.0001 \\
\hline 21.3140 & 0.0000 & 0.0000 & 25.9527 & 0.0000 & 0.0000 \\
\hline
\end{tabular}

$$
K_{2}(\zeta, \xi)=\frac{|\zeta-\xi|}{2 \sqrt{2} \pi} \exp \left(-|\xi|^{2}\right),
$$

and $\ell_{0}=\left[\sqrt{2} \pi \sigma^{2}\left(\rho_{0} / m\right)\right]^{-1}$, where $\sigma$ is the diameter of a molecule.

\section{A. The functions $A, F, F_{d}$, and related constant $\gamma_{2}$}

In the case of the BGK model, the functions $A, F$, and $F_{d}$ are immediately obtained from the definition as

$$
\begin{aligned}
& A(|\zeta|)=|\zeta|^{2}-\frac{5}{2}, F(|\zeta|)=-|\zeta|^{2}+\frac{5}{2}, \\
& F_{d}(|\zeta|)=-\frac{1}{3}\left(|\zeta|^{4}-5|\zeta|^{2}+\frac{15}{4}\right),
\end{aligned}
$$

by which we see $\gamma_{2}=1$. In the case of hard-sphere molecules, they are only known numerically (see Fig. 2). Note that only $F_{d}$ has been computed here; the others are available in the literature and are shown here for easy reference. The value of $\gamma_{2}$ for hard-sphere molecules can also be found in the literature: ${ }^{2,6} \gamma_{2}=1.922284066$.

\section{B. The coefficient $\beta^{(2)}$ of the second-order jump}

In principle, we have to perform a numerical analysis of the problem (20) with $i=2$ to obtain the value of $\beta^{(2)}$. However, by the use of the symmetry of the linearized kinetic equation, we can relate 


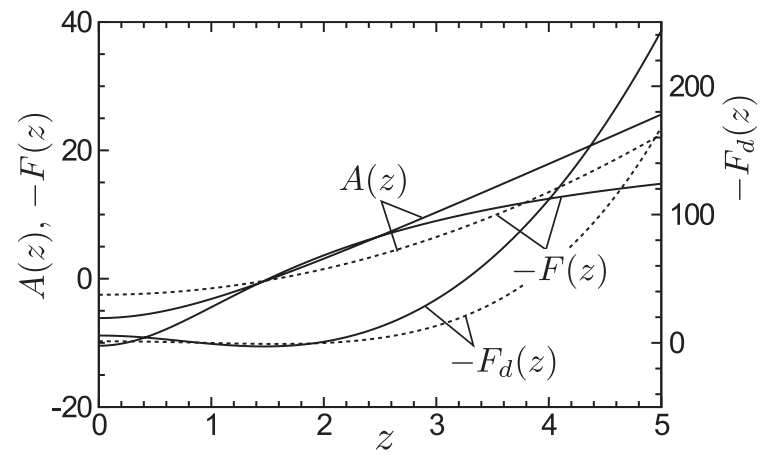

FIG. 2. Functions $A, F$, and $F_{d}$. Solid lines: hard sphere. Dashed lines: BGK.

$\beta^{(2)}$ to quantities occurring in the half-space problem for $\psi^{(1)}$, thereby determining $\beta^{(2)}$ without new computation.

We apply the symmetry relation derived in Ref. 36 to the half-space problems (20) for $\psi^{(1)}$ and $\psi^{(2)}$. The basic idea behind the symmetry relation is that a conservative quantity can be constructed from solutions of two independent problems. (This is a basic principle that can be naturally extended to time-dependent problems. ${ }^{37}$ ) We briefly explain it here. If we multiply Eq. (20a) for $\psi^{(1)}$ by $\psi^{(2)}(\eta,-\zeta)$ and take its moment, we find that $\left\langle\zeta_{1} \psi^{(2)}(\eta,-\zeta) \psi^{(1)}(\eta, \zeta)\right\rangle$, the "flux" of the constructed conservative quantity $\left\langle\psi^{(2)}(\eta,-\zeta) \psi^{(1)}(\eta, \zeta)\right\rangle$, is constant in $\eta$ and thus vanishes because of condition (20c) at infinity. Then, by using the condition (20b) at $\eta=0$, we obtain

$$
\frac{5 \gamma_{2}}{4} \beta^{(2)}=\frac{2}{3} I_{6}(A F)+\frac{5}{2} I_{4}\left(A F_{d}\right)-\left.\left\langle\zeta_{1}\left[F_{d}-\frac{1}{3}\left(|\zeta|^{2}-3 \zeta_{1}^{2}\right) F\right] \psi^{(1)}\right\rangle\right|_{\eta=0} .
$$

To simplify the last term on the right-hand side further, we consider Eq. (20a) for $\psi^{(1)}$ multiplied by $F_{d}-\frac{1}{3}\left(|\zeta|^{2}-3 \zeta_{1}^{2}\right) F$ and take its moment. Then, we obtain

$$
\begin{aligned}
\frac{d}{d \eta}\left\langle\zeta_{1}\left[F_{d}-\frac{1}{3}\left(|\zeta|^{2}-3 \zeta_{1}^{2}\right) F\right] \psi^{(1)}\right\rangle & =\left\langle\psi^{(1)} \mathcal{L}\left[F_{d}-\frac{1}{3}\left(|\zeta|^{2}-3 \zeta_{1}^{2}\right) F\right]\right\rangle \\
& =-\frac{5 \gamma_{2}}{6}\left\langle\left(|\zeta|^{2}-\frac{3}{2}\right) \psi^{(1)}\right\rangle+\left\langle\zeta_{1}^{2} A \psi^{(1)}\right\rangle,
\end{aligned}
$$

where we have used the self-adjointness of $\mathcal{L}$ and the definitions of $F$ and $F_{d}$. We can show that the last term $\left\langle\zeta_{1}^{2} A \psi^{(1)}\right\rangle$ vanishes by the result of two integrations with respect to $\eta$ : one is Eq. (20a) for $\psi^{(1)}$ multiplied by $\zeta_{1} A$ and the other is that multiplied by $\zeta_{1}\left(|\zeta|^{2}-\frac{5}{2}\right)$. Thus, from Eq. (22), we obtain

$$
\left.\left\langle\zeta_{1}\left[F_{d}-\frac{1}{3}\left(|\zeta|^{2}-3 \zeta_{1}^{2}\right) F\right] \psi^{(1)}\right\rangle\right|_{\eta=0}=\frac{5 \gamma_{2}}{4} \int_{0}^{\infty} \Theta^{(1)} d \eta,
$$

and finally arrive at the relation

$$
\beta^{(2)}=\frac{8}{15 \gamma_{2}}\left[I_{6}(A F)+\frac{15}{4} I_{4}\left(A F_{d}\right)\right]-\int_{0}^{\infty} \Theta^{(1)} d \eta .
$$

In the case of the BGK model, $I_{6}(A F)=-9 / 4, I_{4}\left(A F_{d}\right)=-1 / 3$, and $\int_{0}^{\infty} \Theta^{(1)} d \eta=-0.4391$. In the case of hard-sphere molecules, $I_{6}(A F)=-7.4839567, I_{4}\left(A F_{d}\right)=-1.7512934$, and $\int_{0}^{\infty} \Theta^{(1)} d \eta$ $=-0.7184$. Thus, we obtain

$$
\beta^{(2)}=-1.4276(\mathrm{BGK}),-3.1801 \text { (hard sphere). }
$$


TABLE II. Knudsen-layer functions $\Omega^{(2)}$ and $\Theta^{(2)}$.

\begin{tabular}{cccccc}
\hline \hline & Hard sphere & & & BGK & \\
\cline { 4 - 5 }$\eta$ & $-\Omega^{(2)}$ & $\Theta^{(2)}$ & $\eta$ & $-\Omega^{(2)}$ & $\Theta^{(2)}$ \\
\hline 0.0000 & 1.4907 & 2.2601 & 0.0000 & 0.6745 & 1.0345 \\
0.0033 & 1.4743 & 2.2335 & 0.0101 & 0.6546 & 1.0045 \\
0.1142 & 1.2440 & 1.8550 & 0.1499 & 0.5309 & 0.8105 \\
0.4091 & 0.9400 & 1.3584 & 0.5021 & 0.3797 & 0.5703 \\
0.9183 & 0.6507 & 0.8994 & 0.9951 & 0.2644 & 0.3889 \\
1.4994 & 0.4547 & 0.6019 & 1.4977 & 0.1924 & 0.2777 \\
2.0259 & 0.3375 & 0.4318 & 1.9924 & 0.1446 & 0.2054 \\
2.4811 & 0.2641 & 0.3291 & 2.4158 & 0.1149 & 0.1611 \\
2.8272 & 0.2204 & 0.2696 & 3.0859 & 0.0814 & 0.1121 \\
3.1150 & 0.1901 & 0.2292 & 3.7057 & 0.0601 & 0.0816 \\
3.6779 & 0.1432 & 0.1684 & 4.0319 & 0.0515 & 0.0694 \\
4.1865 & 0.1114 & 0.1284 & 4.3158 & 0.0451 & 0.0604 \\
4.6706 & 0.0881 & 0.0997 & 4.7026 & 0.0378 & 0.0502 \\
5.0001 & 0.0752 & 0.0842 & 5.1286 & 0.0312 & 0.0412 \\
5.6738 & 0.0546 & 0.0599 & 5.9037 & 0.0222 & 0.0289 \\
6.4526 & 0.0379 & 0.0408 & 6.5723 & 0.0167 & 0.0215 \\
7.0725 & 0.0285 & 0.0302 & 7.1957 & 0.0129 & 0.0164 \\
7.5226 & 0.0231 & 0.0243 & 7.6019 & 0.0109 & 0.0138 \\
8.0248 & 0.0184 & 0.0192 & 8.1849 & 0.0086 & 0.0108 \\
8.5346 & 0.0146 & 0.0151 & 8.8196 & 0.0066 & 0.0083 \\
9.0048 & 0.0118 & 0.0121 & 9.5114 & 0.0051 & 0.0063 \\
10.0146 & 0.0075 & 0.0076 & 11.9922 & 0.0020 & 0.0024 \\
14.5460 & 0.0010 & 0.0010 & 14.0616 & 0.0009 & 0.0011 \\
20.0515 & 0.0001 & 0.0001 & 20.4253 & 0.0001 & 0.0001 \\
25.0557 & 0.0000 & 0.0000 & 27.7493 & 0.0000 & 0.0000 \\
\hline \hline & & & & &
\end{tabular}

\section{Direct numerical solution of the half-space problem for $\psi^{(2)}$}

In order to obtain $\Omega^{(2)}$ and $\Theta^{(2)}$, we carried out a numerical computation of the problem (20) for $\psi^{(2)}$ by a finite-difference method both for the BGK model and hard-sphere molecules. For the hard-sphere collision integral, we adopted the method first devised in Ref. 32 and applied later to various fundamental problems, including those for gas mixtures (see Refs. 35, 38, and 39, and the references therein). In the numerical computation, we seek the solution $\psi^{(2)}$ as a function of $\eta$, $\zeta_{1}$, and $\zeta_{\rho}\left(\equiv \sqrt{\zeta_{2}^{2}+\zeta_{3}^{2}}\right)$, thanks to the spherical symmetry of $\mathcal{L}$. We have used the grid system in Ref. 38 with more grid points (roughly speaking, about 50\% increase in $\zeta_{1}$ and almost double in $\eta$ and $\zeta_{\rho}$ ) (Footnote 40) and have adopted a third-order finite-difference scheme as in Ref. 39. The computation for the BGK model used even more grid points in $\zeta_{1}$ and $\zeta_{\rho}$.

Table II and Fig. 3 show $\Omega^{(2)}$ and $\Theta^{(2)}$. In the figure, the profiles of $\Omega^{(1)}$ and $\Theta^{(1)}$ are also shown for reference.

The value of $\beta^{(2)}$ was also obtained directly in the computation as

$$
\beta^{(2)}=-1.4276(\mathrm{BGK}),-3.1800 \text { (hard sphere). }
$$

The values in Eqs. (24) and (25) agree to four or five digits. This comparison is an indirect measure of computational accuracy.

In Fig. 4, we show the profiles of macroscopic quantities $\tau$ and $\omega$ of the original two-surface problem, i.e., Eqs. (21b) and (21c), for $\varepsilon=0.01,0.05$, and 0.1 . The profiles look highly dependent on the molecular model. This is, however, mainly due to the fact that the relation between the mean free path of a molecule and the thermal conductivity of the gas depends on the molecular model. To account for this, we take the thermal conductivity of the gas as the basic parameter instead of 


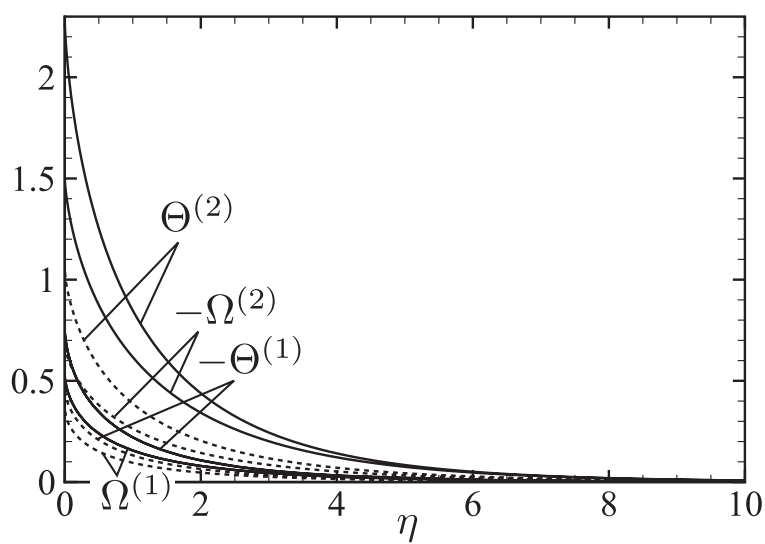

FIG. 3. Knudsen-layer functions $\Omega^{(2)}$ and $\Theta^{(2)}$. Solid lines: hard sphere. Dashed lines: BGK. The profiles of $\Omega^{(1)}$ and $\Theta^{(1)}$ are also shown for reference.

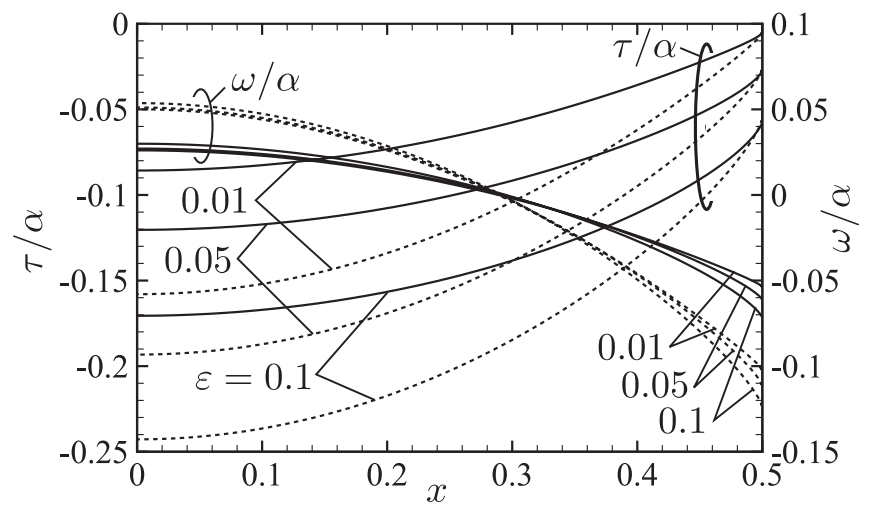

FIG. 4. Profiles of $\tau / \alpha$ and $\omega / \alpha$ in the right half of the gap between the plates [see Eq. (21)] for $\varepsilon=0.01,0.05$, and 0.1. Solid lines: hard sphere. Dashed lines: BGK.

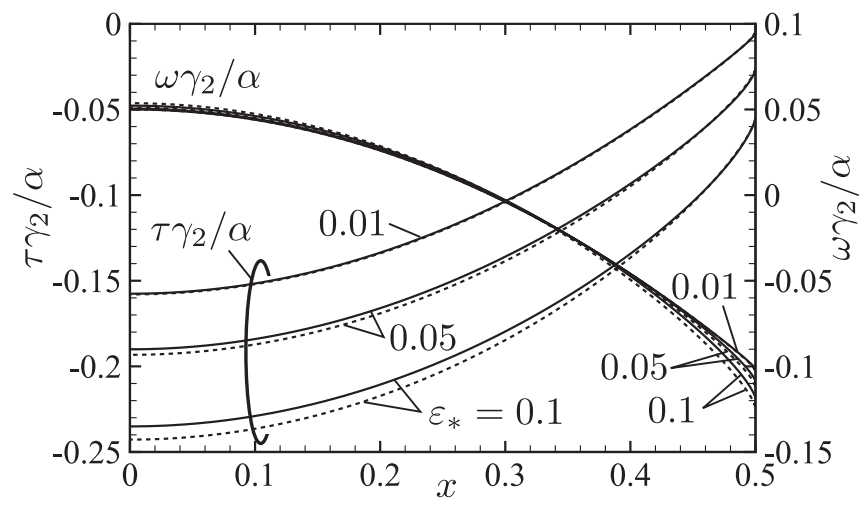

FIG. 5. Profiles of $\tau \gamma_{2} / \alpha$ and $\omega \gamma_{2} / \alpha$ in the right half of the gap between the plates for $\varepsilon *=0.01,0.05$, and 0.1 . Solid lines: hard sphere. Dashed lines: BGK.

the mean free path, introduce $\varepsilon^{*}=\gamma_{2} \varepsilon$, and plot $\tau \gamma_{2} / \alpha$ and $\omega \gamma_{2} / \alpha$ for the same values of $\varepsilon *$. The resulting profiles become less dependent on the molecular model (Fig. 5).

Finally, in Fig. 6 we compare the profile of $\tau$ with that obtained by RHTA for $\mathrm{Kn}=0.05$ and 0.1 for hard-sphere molecules. The two profiles agree quite well; the difference is not visible in the figure. This is probably due to the fact that our asymptotic solution (21) is valid up to any order of $\varepsilon$. 


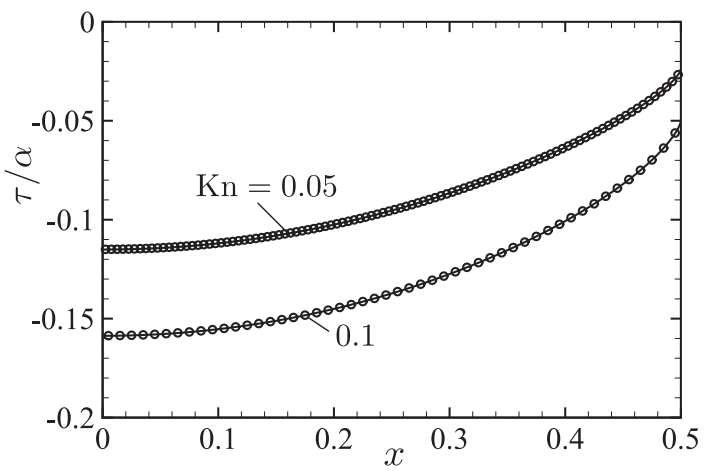

FIG. 6. Comparisons of the profiles of $\tau / \alpha$ with that obtained by Radtke, Hadjiconstantinou, Takata, and Aoki (RHTA) for $\mathrm{Kn}=0.05$ and 0.1 (hard sphere). Solid lines: Eq. (21b). Open circles: RHTA.

\section{CONCLUSION}

We have investigated the behavior of a slightly rarefied monatomic gas between two parallel plates whose temperature grows slowly and linearly in time. To be more precise, we studied the gas behavior that is realized after a long time has passed from some initial state.

We first showed that this problem is equivalent to a boundary-value problem for the steady linearized Boltzmann equation with a volumetric heating source, recently studied by Radtke, Hadjiconstantinou, Takata, and Aoki who focus on the parabolic temperature profile and related second-order jump. Our formulation gives a natural interpretation to the heating source and shows that the nonzero second derivative of temperature and related second-order jump is induced by a mechanism which is not covered by the general theory of slip flow for steady problems. As a result, naive application of the second-order jump condition for steady problems to unsteady problems can produce incorrect results. Further discussion of the differences between the two cases can be found in the paper by RHTA.

We also carried out a systematic asymptotic analysis for small Knudsen numbers. Analysis of the Knudsen-layer correction shows that the second-order jump of temperature is indeed determined by a new half-space problem which does not occur in the general theory of slip flow for steady problems. ${ }^{2,6,11,13}$ This explains why the jump coefficient observed by RHTA does not agree with any of the jump coefficients in the existing theory. The newly found temperature-jump does not influence the instantaneous heat transfer to the walls, at least up to the second order in the present one-dimensional problem, because its associated Knudsen-layer problem for $\psi^{(2)}$ does not have an inhomogeneous term in Eq. (20a) and thus gives no additional contribution to the heat flow. (The heat flux to the wall is always equal to the heating/cooling rate integrated over the domain size and is correctly captured even without knowledge of the first-order slip coefficient.) The theoretical treatment presented here can be straightforwardly extended to gas mixtures with qualitatively similar results.

Finally, we carried out numerical computations to determine the coefficient of the new secondorder temperature jump and the structure of the related Knudsen layer for the BGK model and hard-sphere molecules. The jump coefficient was also calculated by the use of a symmetry relation. The values obtained by the two different methods agree within our estimates of the numerical accuracy of the computations. Moreover, the present asymptotic solution for the behavior of the gas agrees well with the results that RHTA have obtained by the low variance stochastic method. ${ }^{23-26}$

\section{ACKNOWLEDGMENTS}

The present work is partially supported by JSPS KAKENHI (Nos. 23360083 and 23360048). The authors thank Masashi Oishi for his help in recomputing $\psi^{(1)}$ for the BGK model. 
${ }^{1}$ C. Cercignani, The Boltzmann Equation and Its Applications (Springer, New York, 1988).

${ }^{2}$ Y. Sone, Kinetic Theory and Fluid Dynamics (Birkhäuser, Boston, MA, 2002) (as of December 2011, supplementary notes and errata are available at http://hdl.handle.net/2433/66099).

${ }^{3}$ G. Karniadakis, A. Beskok, and A. Aluru, Microflows and Nanoflows (Springer, New York, 2005).

${ }^{4}$ H. Struchtrup, Macroscopic Transport Equations for Rarefied Gas Flows (Springer-Verlag, Berlin, 2005).

${ }^{5}$ C. Cercignani, Slow Rarefied Flows (Birkhäuser, Boston, MA, 2006).

${ }^{6}$ Y. Sone, Molecular Gas Dynamics (Birkhäuser, Boston, MA, 2007) (as of December 2011, supplementary notes and errata are available at http://hdl.handle.net/2433/66098).

${ }^{7}$ D. Hilbert, "Begründung der kinetischen Gastheorie," Math. Ann. 72, 562 (1912).

${ }^{8}$ S. Chapman and T. G. Cowling, The Mathematical Theory of Non-uniform Gases, 3rd ed. reprint (Cambridge University Press, Cambridge, England, 1995).

${ }^{9}$ H. Grad, "Asymptotic theory of the Boltzmann equation," Phys. Fluids 6, 147 (1963).

${ }^{10} \mathrm{H}$. Grad, "Singular and nonuniform limits of solutions of the Boltzmann equation," in Transport Theory, edited by R. Bellman, G. Birkhoff, and I. Abu-Shumays (American Mathematical Society, Providence, 1969), pp. 269-308.

${ }^{11}$ Y. Sone, "Asymptotic theory of flow of rarefied gas over a smooth boundary I," in Rarefied Gas Dynamics, edited by L. Trilling and H. Y. Wachman, (Academic, New York, 1969), Vol. 1, pp. 243-253.

${ }^{12}$ Y. Sone, "Asymptotic theory of flow of rarefied gas over a smooth boundary II," in Rarefied Gas Dynamics, edited by D. Dini, (Edtrice Tecnico Scientifica, Pisa, 1971), Vol. 2, pp. 737-749.

${ }^{13}$ Y. Sone and K. Aoki, "Steady gas flows past bodies at small Knudsen numbers-Boltzmann and hydrodynamic systems," Transp. Theory Stat. Phys. 16, 189 (1987).

${ }^{14}$ A. DeMasi, R. Esposito, and J. Lebowitz, "Incompressible Navier-Stokes and Euler limits of the Boltzmann equation," Commun. Pure Appl. Math. 42, 1189 (1989).

${ }^{15}$ F. Golse and L. Saint-Raymond, "The Navier-Stokes limit of the Boltzmann equation for bounded collision kernels," Invent. Math. 155, 81 (2004).

${ }^{16}$ F. Golse, "From the kinetic theory of gases to continuum mechanics," in 27th International Symposium on Rarefied Gas Dynamics, 2010 AIP Conf. Proc. 1333, edited by D. A. Levin, I. J. Wysong, and A. L. Garcia (AIP, Melville, 2011), pp. 15-27 (2011).

${ }^{17}$ G. A. Radtke, "Efficient simulation of molecular gas transport for micro- and nanoscale applications," Ph.D. dissertation (Massachusetts Institute of Technology, 2011).

${ }^{18}$ C. Aubert and S. Colin, "High-order boundary conditions for gaseous flows in rectangular microducts," Microscale Thermophys. Eng. 5, 41 (2001).

${ }^{19}$ J. Maurer, P. Tabeling, P. Joseph, and H. Willaime, "Second-order slip laws in microchannels for helium and nitrogen," Phys. Fluids 15, 2613 (2003).

${ }^{20}$ S. Colin, P. Lalonde, and R. Caen, "Validation of a second-order slip flow model in rectangular microchannels," Heat Transfer Eng. 25, 23 (2004).

${ }^{21}$ N. Xiao, J. Elsnab, and T. Ameel, "Mictotube gas flows with second-order slip flow and temperature boundary conditions," Int. J. Therm. Sci. 48, 243 (2009).

${ }^{22} \mathrm{C}$. Cercignani and S. Lorenzani, "Variational derivation of second-order slip coefficients on the basis of the Boltzmann equation for hard-sphere molecules," Phys. Fluids 22, 062004 (2010).

${ }^{23}$ T. M. M. Homolle and N. G. Hadjiconstantinou, "Low-variance deviational simulation Monte Carlo," Phys. Fluids 19, 041701 (2007).

${ }^{24}$ T. M. M. Homolle and N. G. Hadjiconstantinou, "Low-variance deviational simulation Monte Carlo for Boltzmann equation," J. Comput. Phys. 226, 2341 (2007).

${ }^{25}$ G. A. Radtke and N. G. Hadjiconstantinou, "Variance-reduced particle simulation of the Boltzmann transport equation in the relaxation-time approximation," Phys. Rev. E 79, 056711 (2009).

${ }^{26}$ G. A. Radtke, N. G. Hadjiconstantinou, and W. Wagner, "Low-noise Monte Carlo simulation of the variable hard-sphere gas," Phys. Fluids 23, 030606 (2011).

${ }^{27}$ P. L. Bhatnagar, E. P. Gross, and M. Krook, "A model for collision processes in gases. I. Small amplitude processes in charged and neutral one-component systems," Phys. Rev. 94, 511 (1954).

${ }^{28}$ P. Welander, "On the temperature jump in a rarefied gas," Ark. Fys. 7, 507 (1954).

${ }^{29}$ N. B. Maslova, "The Kramers problem in the kinetic theory of gases," USSR Comput. Math. Math. Phys. 22, 208 (1982).

${ }^{30}$ C. Bardos, R. E. Caflisch, and B. Nicolaenko, "The Milne and Kramers problems for the Boltzmann equation of a hard sphere gas," Commun. Pure Appl. Math. 39, 323 (1986).

${ }^{31}$ F. Coron, F. Golse, and C. Sulem, "A classification of well-posed kinetic layer problems," Commun. Pure Appl. Math. 41, 409 (1988).

${ }^{32}$ Y. Sone, T. Ohwada, and K. Aoki, "Temperature jump and Knudsen layer in a rarefied gas over a plane wall: Numerical analysis of the linearized Boltzmann equation for hard-sphere molecules," Phys. Fluids A 1, 363 (1989).

${ }^{33}$ C. E. Siewert, "Viscous-slip, thermal-slip, and temperature-jump coefficients as defined by the linearized Boltzmann equation and the Cercignani-Lampis boundary condition," Phys. Fluids 15, 1696 (2003).

${ }^{34}$ S. K. Loyalka, "Slip and jump coefficients for rarefied gas flows: Variational results for Lennard-Jones and n(r)-6 potentials," Physica A 163, 813 (1990).

${ }^{35}$ S. Takata, K. Aoki, S. Yasuda, and S. Kosuge, "Temperature, pressure, and concentration jumps for a binary mixture of vapors on a plane condensed phase: Numerical analysis of the linearized Boltzmann equation," Phys. Fluids 18, 067102 (2006).

${ }^{36}$ S. Takata, "Symmetry of the linearized Boltzmann equation and its application," J. Stat. Phys. 136, 751 (2009).

${ }^{37}$ S. Takata, "Symmetry of the unsteady linearized Boltzmann equation in a fixed bounded domain," J. Stat. Phys. 140, 985 (2010). 
${ }^{38}$ S. Takata, S. Yasuda, S. Kosuge, and K. Aoki, "Numerical analysis of thermal-slip and diffusion-slip flows of a binary mixture of hard-sphere molecular gases," Phys. Fluids 15, 3745 (2003).

${ }^{39} \mathrm{~S}$. Yasuda, S. Takata, and K. Aoki, "Numerical analysis of the shear flow of a binary mixture of hard-sphere gases over a plane wall," Phys. Fluids 16, 1989 (2004).

${ }^{40}$ We have applied to $\eta, \zeta_{1}$, and $\zeta_{\rho}$ the grid system for $x_{1}, \zeta_{1}^{\alpha}$, and $\zeta_{\rho}^{\alpha}$ generated by Eqs. (52a) - (53c) of Ref. 38 with $\hat{m}^{\alpha}=1$. The present numerical data were obtained by the following setting of grid parameters: $\bar{N}_{x}=600, N_{x}=700, \bar{Z}_{1}=\bar{Z}_{\rho}=5$, $\bar{N}_{1}=N_{1}=32$, and $\bar{N}_{\rho}=N_{\rho}=36$. By the present choice of $\bar{N}_{x}$ and $N_{x}$ the $\eta$-space is truncated at $\eta \simeq 35$. 